\title{
Effectual Clustering and Node Placement with Differential Evolution Particle Swarm Optimization using Markov Chain Clustering in FANET

\author{
B. Mahalakshmi, S.Ranjitha Kumari
}

\begin{abstract}
Flying ad hoc network (FANET) comprises of multiple unmanned aerial vehicles (UAVs) which is effectual technology for future generation. In this investigation, the specific way for constructing a FANET topology using clustering technique to achieve end-to-end communication is elaborated. For this purpose, an application that uses the meta-heuristics approach for cluster analysis is anticipated. Specifically, the parameters of differential evolution (DE) and particle swarm optimization (PSO) have gained the attention and extensive popularity in various communities based on its working effectiveness in resolving complex combinational optimization crisis. Thus, hybrid model of DE and PSO based Markov Chain Clustering Model (MCCM) is designed in this investigation to analyse the problems of clustering in FANET and reliability parameters are examined. The proposed (DEPSO-MCM) model is to enhance search capability and to attain superior flexibility in forming nodes cluster. Empirical outcomes demonstrate DEPSOMCM based clustering algorithm attains superior performance in number of epochs to acquire fitness function effectually. The simulation was carried out in NS-2 simulator, the outcomes based on the simulation shows that the proposed method works effectually and shows better trade-off than the existing techniques to provide a meaningful clustering.

Index Terms- Unsupervised classification; Particle swarm optimization; Differential evolution; Markov Chain Model; Reliability
\end{abstract}

\section{INTRODUCTION}

An unmanned aerial vehicle (UAV) is flying device which may not necessitate any onboard pilots. UAV specifically equipped with radio communication modules that can be controlled in manual way by operators in ground control station or autonomous way by flight program. UAV can guarantee pilot safety that can be extensively utilized in military applications. In specific, the most distinctive FANET characteristics is UAVs passes over the height by speeds based on applications. However other networks, due to higher mobility nature of UAVs cause topological changes rapidly. As well, UAVs location may be changed for every second, so that links between UAVs are destroyed and established intermittently as in Fig 1 . Therefore, there are numerous works based on topology managements for FANETs to deal with this crisis.

Revised Manuscript Received on October 30, 2019.

* Correspondence Author

B. Mahalakshmi*, Research Scholar, School of Computer Studies, Rathnavel Subramaniam College of Arts and Science, Sulur, Coimbatore.

Dr.S.Ranjitha Kumari, Associate Professor, School of Computer Studies, Rathnavel Subramaniam College of Arts and Science, Sulur, Coimbatore

(C) The Authors. Published by Blue Eyes Intelligence Engineering and Sciences Publication (BEIESP). This is an open access article under the CC BY-NC-ND license (http://creativecommons.org/licenses/by-nc-nd/4.0/)
By examining various investigations UAV placement problems with pre-determined MANETs improve network connectivity. With pre-configured MANETs, problems on placing UAVs to reduced communication length. As well, problem to make disconnected network to be done by placing least amount of UAV is also studied. When networks are damaged owing to certain unpredicted disasters, problems to place UAVs to establish connectivity are also analysed.

One most significant and primitive clustering activities is termed as unsupervised classification or data analysis, that tries to investigate information by partitioning finite data, with lesser or no ground truth discrete set of hidden data structures. In specific, clustering is designed as optimization crisis by minimizing or maximizing pre-defined function, thus data objects are similar to others in cluster than others in various clusters. Even though, certain optimal partition is accomplished by determining possibilities, brute-forcing is infeasible while implementation expensive computation.

General alternative to utilize heuristic algorithms, for instance, general utilization of k-means to hunt optimal solutions are with sensible computational burdens. Moreover, hill-climbing based clustering procedures suffer disadvantages and it can simply strike in its local minima devoid of global optimality. For this cause, meta-heuristics like evolutionary algorithms, DE, ACO, PSO offer more effectual technique to explore clustering solution space. In specific, clustering DE and PSO along with Markov model has gained increasing popularity, attention, and effort from variety of research communities based on implementation and execution effectually in resolving complex combinatorial optimization crisis.

Merwe and Engelbrecht [1] utilized particle swarm optimization to attain pre-specified amount of centroids and also recommended the use of k-means outcomes to particle initialization. In [2], author also considers the combination of k-means and PSO in clustering documents context but clustering outcomes are preliminary seeds to process partition. PSO based implementation invariant of $\mathrm{k}$-means is termed as k-harmonic and c-means algorithm as in [3] and [4] correspondingly to alleviate user-specific dependence over number of clusters. In [5] the author defined every particle as vector with length equal to maximum cluster amount, which is much simpler for evaluation.

Every bit in particle indicates whether the respective cluster centroid is in co-operated in clustering solution or not. In [6] author shows that a PSO designed as a combination of a set of cluster centroids and activation threshold, therefore extending its limitation of above techniques that consider cluster centroids in every iteration. 
In [7] the investigator compared PSO and DE performance in robustness and precision of clustering algorithms and suggested DE on empirical outcomes. In [8], the investigator followed a similar encoding scheme as its PSO based clustering enhanced parameter to speed up its convergence.

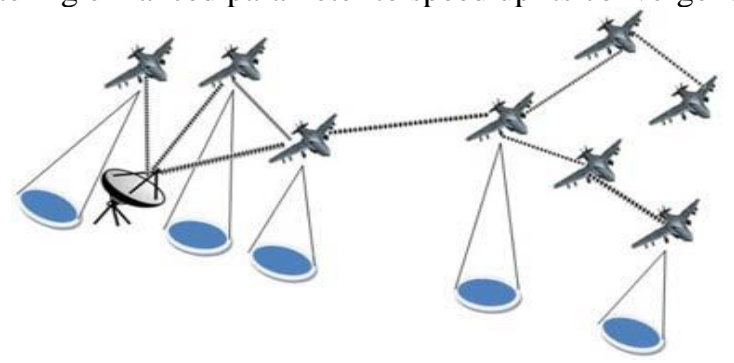

Fig 1: Architectural diagram of FANET

Here, this work investigated the applications of hybrid DE and PSO along with the mathematical modelling which is known as DEPSO-MCCM, in partitional clustering, determining that, DEPSO-MCCM's constant performance in resolving numerous optimization problems [9]. As well, an effectual hybridization of diverse evolutionary computational approach can also enhance capability and attains superior flexibility in exploring problem space [10], therefore attaining efforts in a combination of optimization and evolution computation. PSO shows a search process on cognitive components. Complete swarm attempts to use global best, therefore enhancing own position. However, for particle, i.e., global best, velocity based weighted. DEPSO merges DE and PSO procedure to include PSO diversity, therefore maintaining particles from falling to local minimum. Integrating mathematical Markov chain model with DEPSO is to enhance the placement of particles and its connectivity to next state provides empirical outcomes that attain better performance based on a number of periods needed to acquire pre-specified cut-off value of fitness function than DE or PSO. Experimental outcome demonstrates that the proposed model shows better trade-off in contrast to the existing methods in finding effectual clustering solutions.

The below-given factors are the key contributions of the proposed DEPSO-MCCM:

- The proposed method is an initial tries where certain DE or PSO based Markov chain clustering model is formulated for effectual node connectivity in FANET.

- $\quad$ At first, the clustering technique based search capability of DE and next is based on search capability of PSO and state detection using Markov chain model.

- $\quad$ The above-mentioned methods are deployed with point symmetry-based distance for assigning points between diverse clusters.

- $\quad$ The combined DE and PSO based clustering techniques are capable to identify clusters and offer nodes partitioning.

- Partitioning measures are provided in reliability, efficiency used as optimization objective.

- Outcomes based on meta-heuristic model depicts performance of DEPSO-MCCM technique is better in contrast to other symmetry-based algorithms.

- $\quad$ Superior performance of DEPSO-MCCM in accuracy is higher than other methods.
- At last, tests are performed to compute statistical importance of results attained.

Remainder of the work is trailed as. Section II depicts existing clustering model together with clustering application. Section III depicts detailed illustration of anticipated DEPSO-MCCM method in reliability and efficiency. Section IV shows numerical results and discussions with respect to DEPSO-MCCM approach. Section V is the brief description of the proposed work and the idea for future extension of the work.

\section{RELATED WORKS}

In 2012, M. S. Fareed, N. Javaid et al, [11] describes that WSNs are extensively equipped to deal with the complex functionalities like aggregation, computation, data fusion, and activities, sensors use its energy effectually to increase network lifetime effectually. As SNs are extremely prone to failure and drainage, therefore re-energizing is needed as already placed SNs die. This issue has ability to damage performance and network stability if energy is not utilized approximately. MATLAB simulation is carried out to compare cluster formation of SEP, DEEC, LEACH. From iterative outcomes, it is determined as DEEC chooses optimal CHs to forward data to BS and to enhance lifetime of network.

In 2016, Hassan El Alami et al, [12] anticipated an approach to widen lifetime and diminish energy utilization in WSNs. To attain this target anticipated approach completely concentrates in the cluster head selection process. EEFL-CH approach chooses cluster head by considering residual energy, expected efficiency and nearness to BS of nodes. From the simulated outcome, it is concluded that anticipated EEFL-CH is effectually and maximizing lifetime of complete network and to attain superior performance in contrast to LEACH protocols.

In 2009, Choon-Sung Nam et al [13], depicted a $\mathrm{CH}$ selection model for uniform cluster sizes in WSNs. This method also presents multi-hop cluster formation technique with a constraint communication nodes range to adjust anticipated model. Again $\mathrm{CHs}$ are re-selected using EACHS considering the distance amongst SNs and CHs and re-organizes cluster with new $\mathrm{CHs}$ generating uniform cluster sizes.

In 2011, Hironori Ando et al, [14] illustrated about power conservation in a wireless sensor network is an extremely significant crisis. Conserving power extends nodes lifetime and network life. Clustering is one amongst the technique for enhancing lifetime of SNs. Clustering approaches organizes nodes into cluster where some nodes function as cluster head and collect data from nodes in clusters. Moreover, cluster head selection is extremely complex when numerous factors are utilized for decisions.

In 2017, Aniji John et al, [15] tested the recital of ES-CHS in a sensor network. Clustering is carried out using uniform clustering. Nodes that have RE superior than average RE of cluster is selected as $\mathrm{CH}$. 
With simulation outcomes, it is complete that ESCHS enhances RE in SNs when contrast to LEACH. Algorithm is simulation for around 2000 rounds. From simulation outcomes in 100 nodes, it is absolute that savings in ESCHS are raised by $10.80 \%$ in contrast to $\mathrm{LEACH}$ and $9.14 \%$ in contrast to D-LEACH. Hence, ESCHS rises lifetime. Furthermore, number of nodes is simulated. However, energy models are not suitable for applications in this algorithm.

In 2018, Chong Han et al, [16] illustrated about heterogeneous network clustering algorithms (CHSES) owing to energy supply is anticipated to resolve constrained battery power that influences the survival time and performance of SNs. Energy around node is transformed into power supply, and nodes are partitioned to nodes with energy self-replenishment and limited nodes. Node dormancy limit is fixed and $\mathrm{CH}$ selection to optimize energy consumption is utilized in accordance with nodes residual energy and self-recharge state of nodes. This approach is sourced on multi-hop routing for transmission. Outcomes demonstrate that anticipated algorithm effectually enlarges network lifetime and enhances energy balance and network stability.

In 2018, Pawan Singh Mehra, [17] illustrated the proliferation of distance from SNs from BS, essential power improves exponentially and if $\mathrm{SN}$ is located at distance from $\mathrm{BS}$ is selected to $\mathrm{CH}$, then it leads to SNs death. Therefore, distance has an essential task in $\mathrm{CH}$ selection. In FBECS, network is partitioned to sub-network and probability is assigned to node as distance separation. FL integration in clustering, anticipated work has shows superior performance with LEACH and BCSA. Stability period of HND has improved. Total information forwarded to BS in FBECS is higher than higher average remnant energy/round.

In 2018, Achyut Shankar et al, [18] offered hybrid GGWSO to improve recital of $\mathrm{CH}$ selection in SNs; therefore networks lifetime can be enhanced. Indeed, the anticipated model is anticipated on delay, distance, security and energy to construct $\mathrm{CH}$ selection in SNs. After performing simulation, performance of anticipated GGWSO was in contrast to traditional algorithms such as ABC, FABC $\mathrm{CH}$ selections. However, diverse levels of risks such as 20, 60, and $100 \%$, and performance were performed by NNE and NAN. Performance of anticipated GGWSO with NAN is 72.09, 19.44\% better than ABC, FABC algorithms where $\mathrm{NNE}$ is superior to $\mathrm{ABC}, 23.39 \%$ superior to FABC, $51.94 \%$ superior to GSO, and $5.86 \%$ superior to GWObased $\mathrm{CH}$ selection techniques whilst security and energyaware network. Therefore, maximized networks lifetime can be attained using GGWSO by offering optimal $\mathrm{CH}$ selection.

In [19], Fang Zhu explained about localization-free routing protocol, termed EERBLC. This protocol attempts to resolve crisis of higher energy consumption, E2E delay, and error rate. An underwater monitoring area is layered, and SNs are clustered at layer. EERBLC in co-operates three phases: transmission routing, cluster formation, maintenance, and cluster update. First, novel unequal cluster technique is appropriate for UWSNs is anticipated to resolve 'hotspot' crisis. EERBLC enhances $\mathrm{CH}$ selection. Multi-objective optimization method is initiated to compute cost value of every candidate's head. In transmission, EERBLC utilizes forwarding ratio and RE of SNs as performance metrics.

In 2018, Adem Fanos Jemal et al, [20] anticipated clusterbased energy-efficient router placement for SNs. In order to equip the anticipated strategy, a k-means is utilized to place routers in procedure of establishing CHs. This work also anticipates fault-tolerant cluster head algorithm to determine secondary cluster head from member nodes owing to proximity and battery budget, when present $\mathrm{CH}$ fails, secondary CHs turns to be cluster head and carries out role of previous cluster head. Performance of anticipated scheme was examined to energy consumption, packet loss, and E2E delay. Based on simulation outcome, it is observed that scheme has attained better performance than LEACH.

\section{PROPOSED METHOD}

This section discusses in detail about clustering the FANET nodes using the anticipated DEPSO based Markov chain clustering model. Here, clustering is performed using DEPSO whereas the placement of nodes for the next successive transmission is evaluated using Markov Chain clustering model as in Fig 2. Illustration of anticipated model is given below:

One of the foremost disadvantages of implementing the differential evolution based particle swarm optimization (PSO) for clustering mobility nodes is particle (node) encoding. With respect to clustering of ' $\mathrm{N}$ ' data objects $A=$ $\left\{a_{1}, a_{2}, \ldots, a_{n}\right\}$ where $a_{n}=\left\{a_{n 1}, a_{n 2}, a_{n 3}, \ldots, a_{n x}\right\}$ into ' $\mathrm{X}$ ' with cluster centroids as $M=\left\{m_{i}\right.$, where $\left.i=1, \ldots X\right\}$. The particles will not directly be encoded to the ' $\mathrm{X}$ ' clusters which denote $z_{i}=\left\{m_{1}, m_{2}, \ldots, m_{k}\right\}$ is termed as centroid sourced representation. Other particle are accessible like partition-based representation, determines every individual in swarm as ' $\mathrm{N}$ ' objects string and ' $\mathrm{i}$ 't' string element specifies cluster number allocated to an $\mathrm{i}^{\text {th }}$ data object. For instance, clustering data object representation into clusters $\left\{x_{1}, x_{3}, x_{5}\right\}\left\{x_{2}, x_{4, x_{7}}\right\}\left\{x_{6}, x_{8}, x_{9}, x_{10}\right\}$ is specified as a string ' 1233211323 '. Moreover, the primary ideas of particle swarm optimization are modified to resolve the situation that candidates are integer vectors.

Primary disadvantage of relating centroid based representation is necessity of determining node clusters to be performed, that may be undesirable in practice. This problem can be resolved using the concept of considering every particle as binary vectors, that is, the length is equal to a maximum amount of clusters, $X \max$, that is, $z_{i}=$ $\left\{z_{i 1}, z_{i 2}, \ldots, Z_{\text {imax }}\right\}, z_{i j} \in\{0,1\}$ for $j=1, \ldots, k_{\max }$. This makes the simpler way of estimating the maximum amount of clusters generated than the actual amount of clusters. Therefore, if $z_{i j}=1$, associating cluster centroid which is choose as clustering solution. As well, $Z_{i j}=0$, associating cluster centroid which is excluded from present solution. It is assumed that, encoding strategies is cluster centroid set is considered for iteration. 
Here, particle representation is anticipated which extends binary particle by representing Here, particle representation is anticipated which extends binary particle by representing $K_{\max }$ set of clusters centroid at time, rather than one. It is provided that maximum amount of clusters $K_{\max }$ and ddimensional cluster centroids set $\left\{m_{i j}, j=1, \ldots, k_{\max }\right\}$, encoded particle is $k_{\max }+k_{\max } * d$ vector, $z_{i}=$ $\left(\tau_{i k \max }, m_{i 1}, \ldots, m_{\text {ikmax }}\right)$ where $\tau_{i j}\left(j=1, \ldots, k_{\max }\right)$ is TH in range of $[0,1]$. TH as control parameter, that evaluates whether cluster centroid is chosen or not.

If 0.5 is superior, cluster is selected; else, cluster turns to an inactive state. For instance, vector (0.57 0.110 .200 .850 .78 (2.9 5.7) (2.5 4.3) (2.9 3.8) (3.5 2.6) (4.2 5.0)) is sample of particle encoding cluster partition in $2 \mathrm{D}$ with maximum cluster amount as 5 .

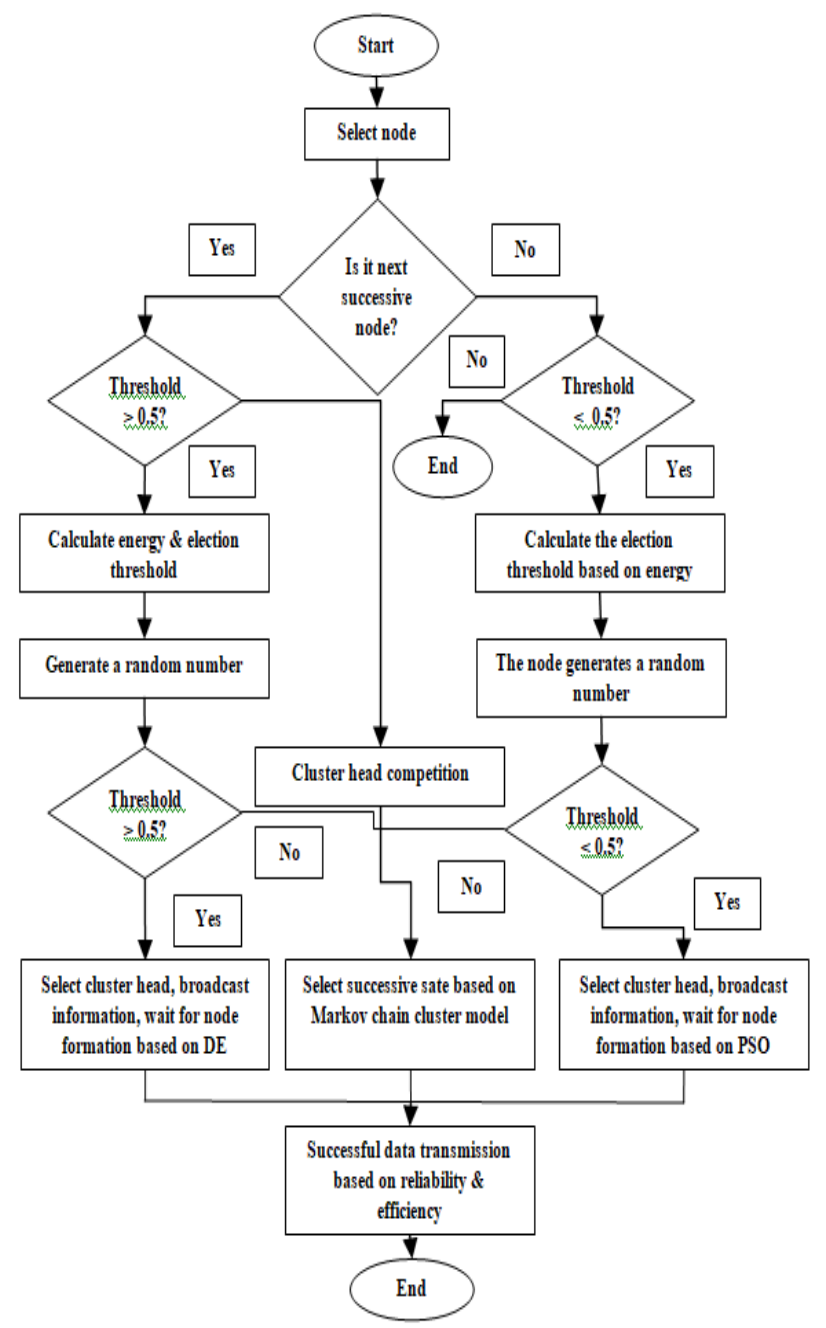

Fig 2: Flowchart of the DEPSO-MCCM approach

Activation thresholds (TH) $0.67,0.95$, and 0.88 specifies $1^{\text {st }}$, $4^{\text {th }}$, and $5^{\text {th }}$ clusters with centroids (1.9 6.7), (4.5 3.6), and (5.2 6.0) are activated. Data objects are allocated with three clusters. Subsequently, two clusters with TH less than 0.5 ( 0.21 and 0.3$)$ is not considered. TH is negative or greater than one and fixed to 0 or 1 , correspondingly. If $\mathrm{TH}$ for certain particle is not greater than 0.5 , then no clusters are activated, thresholds are chosen randomly and re-initialized to 0.5 to 1 to guarantee existence of two clusters. If there are empty cluster, then cluster centroid placement may be too far from boundaries of data set distributions, cluster are deactivated by randomly setting $\mathrm{TH}$ in range of $[0,0.5]$ and validate condition whether minimum amount of clusters (2) is fulfilled.

Subsequent problem associated with DEPSO based clustering is to select fitness function. At present, clustering specifies fitness function to determine quality of cluster. Moreover, validated indices are generally modelled to resolve some data-dependent, selection of diverse indices as fitness function that cause diverse clusters, therefore which affects a finding of meaningful cluster structures. The cluster head selection indices are given below.

Cluster head index attains better performance amongst 30 indices in contrast to Milligan and cooper. The set of ' $N$ ' nodes $A=\left\{a_{1}, \ldots, a_{N}\right\}$ allocated to ' $\mathrm{K}$ ' clusters $C=$ $\left\{C_{1}, \ldots, C_{K}\right\}$ with centroids $m_{i}, i=1, \ldots, K$, cluster head index is provided as in Eq. 1,

Clustered $(K)=\frac{T_{r}\left(S_{B}\right)}{K-1} / \frac{T_{r}\left(S_{W}\right)}{N-K}$

Where, $T_{r}\left(S_{B}\right)=\sum_{i=1}^{k} N_{i} \| m_{i}-\left.m\right|^{2} \quad$ and $\quad T_{r}\left(S_{w}\right)=$ $\sum_{i=1}^{k} \sum_{j=1}^{N_{i}} \quad\left\|X_{j}-m_{i}\right\|^{2}$

The above-mentioned factors are traces of cluster between and scatter matrices correspondingly. Here, $N_{i}$ is number of FANET nodes that belongs to cluster $C_{i}$ and ' $\mathrm{m}$ ' is total mean vector for complete network connectivity. Huge amount of $C H(k)$ provides clustering outcomes with superior quality.

Assume the nodes $x_{j}$ which belongs to cluster $c_{i}$ that posses average distance from all data objects in $c_{i}$ as in Eq.2

$a_{j}=\frac{1}{N_{i}-1} \sum_{i=1, \ldots, N} \| x_{j}-X_{n}||$

For subsequent clusters, $C_{h}, h=1, \ldots, k$ and $h \neq i$, average distance of $x_{j}$ to objects in $C_{h}$ is specified as in Eq.3,

$b_{j}=\min _{h=1, \ldots, k} \frac{1}{N} \sum_{x_{l} \in c_{h}} \| x_{j}-x_{i} \mid$

It is attained that width of $x_{j}$ evaluates how the nodes are clustered as in Eq.4.

$p_{j}=\frac{b_{j}-a_{j}}{\max \left(a_{j}, b_{j}\right)}$

Therefore, positive width specifies corresponding nodes are clustered well, while negative large width determines that the associated objects are clustered wrongly. Node with the positive width zero is not measured as discriminated among clusters. Clustered index is distinct as average positive width over nodes as in Eq.5.

$C=\frac{1}{N} \sum_{j=1}^{N} S_{j}$ 
From the above equation, if ' $\mathrm{C}$ ' value is larger, it specifies the good cluster partition. After the successful formation of the cluster, the data transmission based on successive nodes has to be determined, this is achieved using Markov chain clustering model.

\section{Markov chain clustering model}

In this segment, the anticipated Markov chain clustering model is determined. In this stochastic process $\left\{X_{r}, r=\right.$ $1,2,3, \ldots\}$ is termed as Markov chain, here $X_{r}$ determines nodes position, if it fulfils Markov property [21].

State ' $\mathrm{S}$ ' of Markov chain describes node traversed to original network [22]. For instance, the state created is given below. Conditional probability of next state is based on current state and independent of previous state. Assume $S=$ $\left\{S_{i}, i=1,2,3, \ldots\right\}$, initial state $S_{1}=\{1\}$ of Markov chain comprises of the single source node and the exhausted node or drained node is given as $|S|=\{1,2,3, \ldots,|N|\}$ comprises all nodes in a network and it is not probable to depart, therefore, ' $\mathrm{S}$ ' is finite state space.

Consider a sample network with exhausted state, $S=$ $\{1,2,3,4\}$ comprises all nodes connected to a network, in which the instance state $S_{4}$ of state-space S comprises nodes $\{1,2,3\}$ and connected network components constructed by 1,23 nodes.

Last state comprises network node destination, where Markov model does not progress anymore [23], and it is called assumption ' $\mathrm{i}$ '. The established state comprises traversed nodes of a network, those reached from nodes of previous state, and termed as assumption II. No prior permission to return from last traversed node [24]; moreover, it is probable to wait in current state. Obviously, new state is provided when arc $(I, j) \in A$ is traversed to node ' $\mathrm{i}$ ' in current state and node ' $\mathrm{j}$ ' in next state, and termed as assumption III. As a result, wait states are one of option 1 or option 2 [25].

State-space diagram of established Markov chain model for sample network is constructed. Values on arcs demonstrate wait and transition probabilities.

Transition probabilities satisfy the following conditions:

1) $0<p_{k l}$ for $k=1,2, \ldots,|S|$ and $t=1,2, \ldots,|S|$

2) $\sum_{l} p_{k l}=1$, for $k=1,2, \ldots,|S|$

Transition probabilities are matrix element $\mathrm{P}|\mathrm{S}| \times|\mathrm{S}|$, where $\mathrm{p}_{\mathrm{kl}}$ is $\mathrm{k}^{\text {th }}$ row and $\mathrm{l}^{\text {th }}$ column $\mathrm{P}$, and termed as transition matrix. Algorithm is cast off to acquire transition matrix of Markov chain in network.

1) BEGIN
2) while $\mathrm{CH}<$ cluster1 do
3) if Node[i].state == 'CH' then
4) Node[i].state = 'Node';
5) endif
6) endwhile
7) while $\mathrm{CH}<$ cluster2 do
8) if Node[i].property == 'Markov chain property' then
9) if Node[i].energy > threshold value 0.5
10) predict node energy consumption of nodes;

11) Compute election threshold of node[i] by Equation [];

12) Random state 1 between 0 and 1 ;

13) if $\mathrm{l}<\mathrm{CH}$ threshold then

14) Node[i].state = 'Cluster Head';

15)Transmit Msg to successive state;

16) receive msg from connected $\mathrm{CH}$;

17) Place $\mathrm{CH}$ List with distance;

18) endif

19) endif

20) else if node[i].energy $<$ TH then

21) terminate and do not perform next transmission

22) endif

23) endif

24) else if Node[i].Markov property = 'Ordinary' then

25) if Node[i].energy $>0$ then

26) Compute threshold of Node[i] by Equation [1];

27) Construct random numbers 1 between 0 and 1;

28) if $\mathrm{l}<$ election threshold then

29) Node[i].state = 'CH';

30) Transmit Msg;

31) attain Msg from competition $\mathrm{CH}$;

32) store $\mathrm{CH}$ list with distance as in Eq [2];

33) endif

34) endif

35) else if Node[i].energy then

36) Terminate network;

37) endif

38) endif

39) endwhile

40) while $\mathrm{CH}<$ cluster 3 do

41) if Node[i].state $==$ 'Node' then

42) Choose nearest $\mathrm{CH}$ Sensor[j] from Cluster Head list as in Eq. [3];

43) Node[i].head $=$ Node[j];

44) Transmit node Msg to Node[j];

45) endif

46) else if Node[i].state $==$ ' $\mathrm{CH}$ ' then

47) merge Msg from Cluster Member;

48) store in List;

49) endif

50) endwhile

51) END

\section{NUMERICAL RESULTS AND DISCUSSIONS}

DEPSO-MCCM is executed to validate performance and evaluated with EIPBP and AODV. Simulation is done in NS-2 as all types of optimizations are available and it is easier for implementation. Nodes randomly positioned in $100 * 100 \mathrm{~m}^{2}$. Optimum $\mathrm{CH}$ percentage is taken as $10 \%$. The position of cluster head is maintained in target area to generalize protocol for diverse application. Base station position is determined. Scenarios have been considered as state $1,2,3, .$. for anticipated work. In Scenario 1, nodes are clustered with energy consumption where total nodes count is 100 . In Scenario 2, nodes are provided with energy and total nodes are 200. For comparison, values of EIPBP and AODV are provided with DEPSO-MCCM. Anticipated protocol is executed for normalized outcomes. 
DEPSO-MCCM performance validation is done with overhead, PDR, energy, nodes' delay in each round, successful PDR to BS and average power consumption of network in every round.

\begin{tabular}{|c|c|}
\hline Parameter Description & Value \\
\hline Total nodes & 100 \\
\hline BS & {$[50,50]$} \\
\hline Node ratio & 0.3 \\
\hline Ideal CH & 0.1 \\
\hline Energy consumption & $0.5 \mathrm{~J}$ \\
\hline Energy dissipation & $50 \mathrm{~nJ} / \mathrm{bit}$ \\
\hline Energy consumption & $10 \mathrm{pJ} / \mathrm{bit} / \mathrm{m}^{2}$ \\
\hline Receiver side loss & $50 \mathrm{~nJ} / \mathrm{bit}$ \\
\hline Data transmission & $5 \mathrm{~nJ} / \mathrm{bit}$ \\
\hline \% of energy consumption & $5 \mathrm{~nJ} / \mathrm{bit}$ \\
\hline Packet length & $3000 \mathrm{bit}$ \\
\hline Number of rounds & 3000 \\
\hline
\end{tabular}

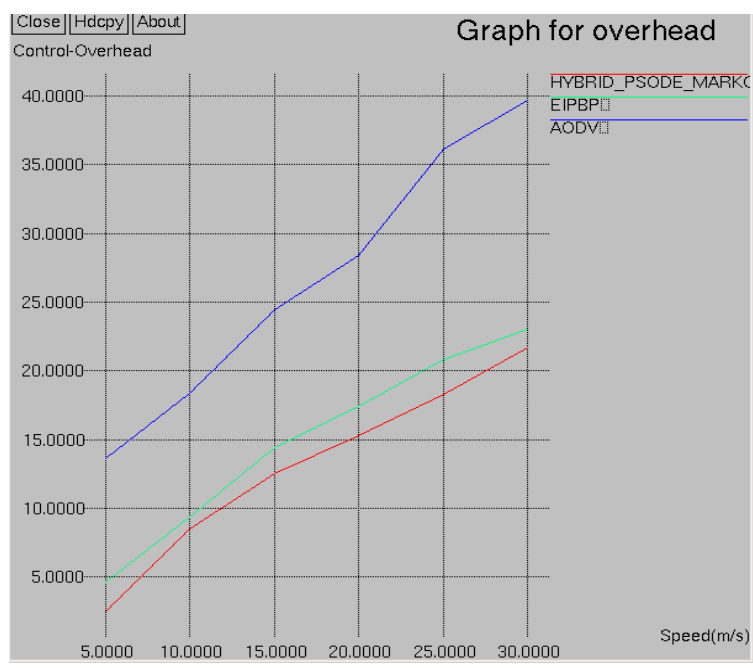

Fig 3: Graphical representation of Mobility based overhead

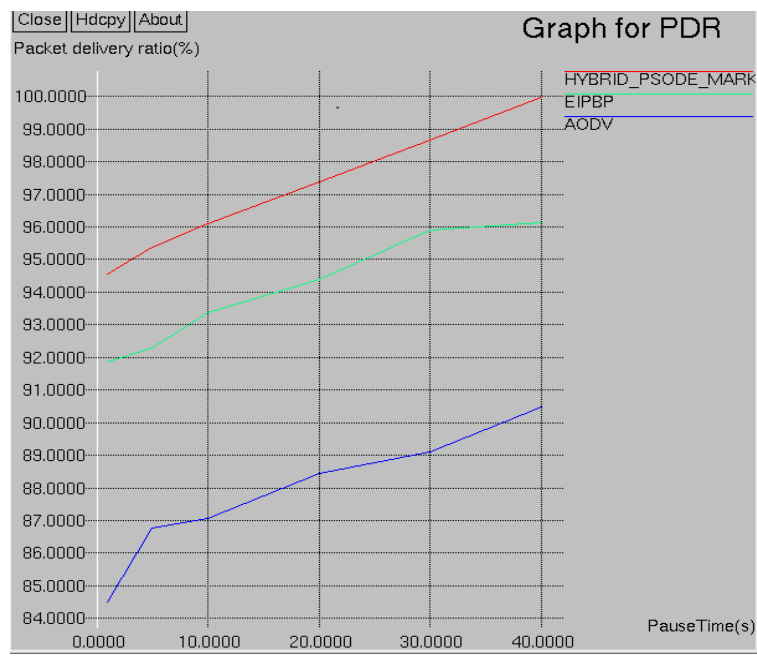

Fig 4: Graphical representation of Mobility based Packet Delivery ratio

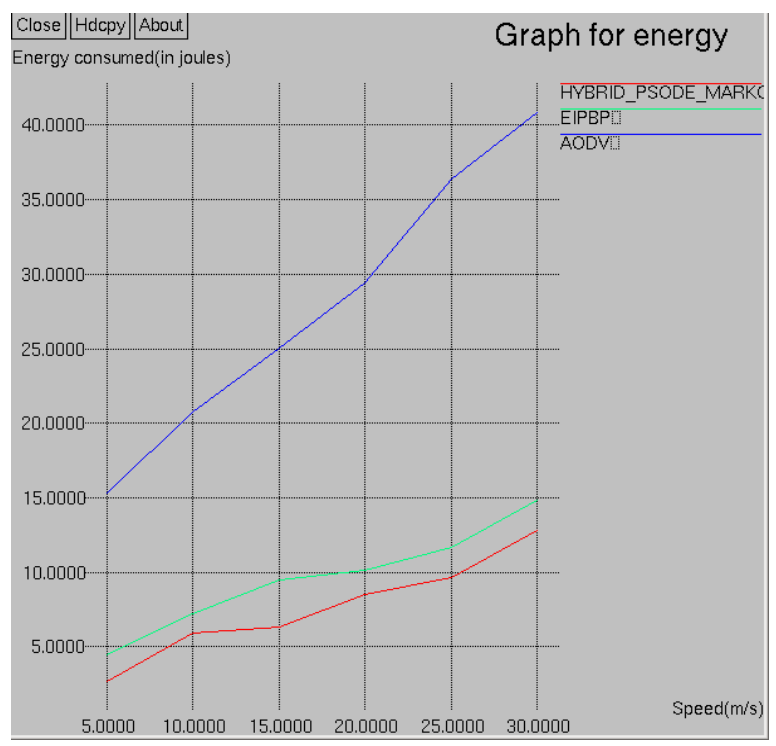

Fig 5: Graphical representation of Mobility based Energy utilization

Figure 3 illustrates graphical representation of DEPSOMCCM approach based on control overhead encountered. Figure 4 depicts graphical representation of PDR based on mobility of FANET nodes into the clustered formed.

Figure 5 depicts graphical representation of energy utilization of nodes connected in network. If the threshold level crosses the boundary higher amount of energy will be consumed due to the larger amount of packet transmission.

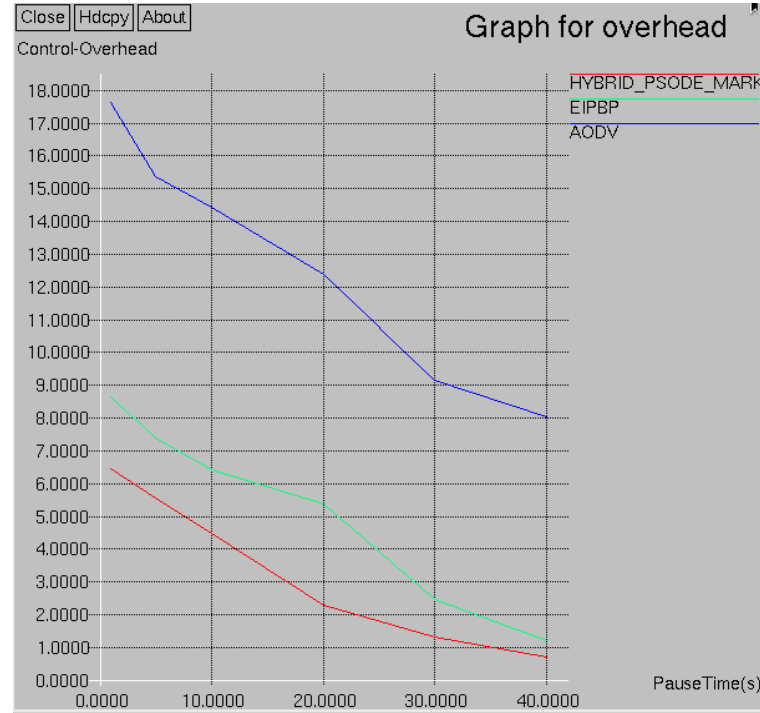

Fig 6: Graphical representation of Pause time-based Overhead 


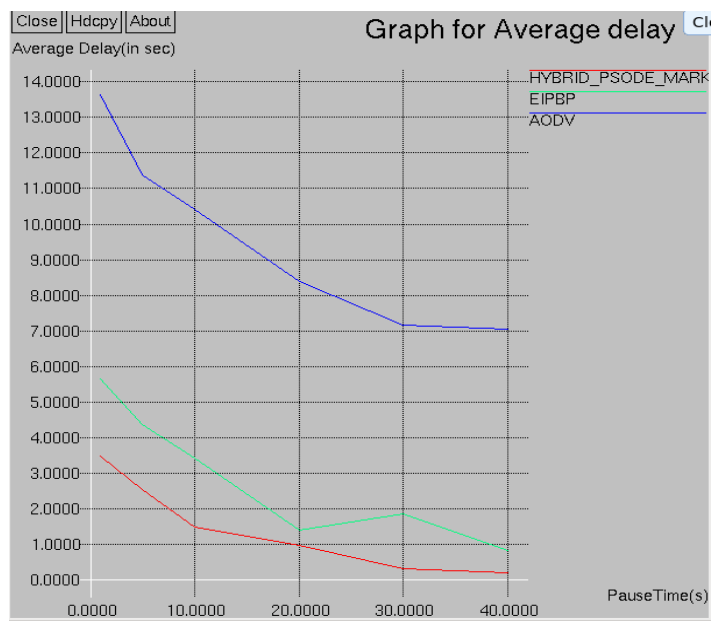

Fig 7: Graphical representation of Pause time based Average delay

Figure 6 shows the graphical representation of the DEPSOMCCM approach based on control overhead encountered in pause time. Figure 7 depicts graphical representation of PDR based on the pause time of FANET nodes into the clustered formed.

Figure 8 shows the graphical representation of energy utilization of nodes connected in network. If the threshold level crosses the boundary higher amount of energy will be consumed due to a larger amount of packet transmission.

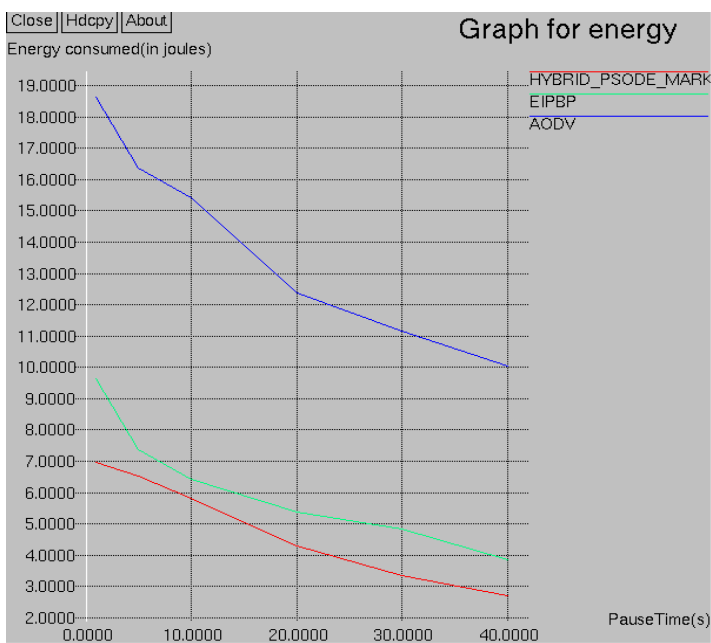

Fig 8: Graphical representation of Pause time-based Energy

Table II: Tabular representation of Mobility based Control overhead

\begin{tabular}{|c|c|c|c|}
\hline Nodes & $\begin{array}{c}\text { DEPSO- } \\
\text { MCCM }\end{array}$ & EIPBP & AODV \\
\hline 5 & 2.47 & 4.65 & 13.65 \\
\hline 10 & 8.54 & 9.38 & 18.38 \\
\hline 15 & 12.49 & 14.42 & 24.42 \\
\hline 20 & 15.28 & 17.39 & 28.39 \\
\hline 25 & 18.33 & 20.85 & 36.15 \\
\hline 30 & 21.69 & 22.99 & 39.72 \\
\hline
\end{tabular}

Table III: Tabular representation of Mobility based Delay

\begin{tabular}{|c|c|c|c|}
\hline Nodes & $\begin{array}{c}\text { DEPSO- } \\
\text { MCCM }\end{array}$ & EIPBP & AODV \\
\hline 5 & 1.34 & 3.49 & 7.49 \\
\hline
\end{tabular}

\begin{tabular}{|c|c|c|c|}
\hline 10 & 7.38 & 9.28 & 14.76 \\
\hline 15 & 9.67 & 11.37 & 25.07 \\
\hline 20 & 12.48 & 13.83 & 29.43 \\
\hline 25 & 15.33 & 16.18 & 34.57 \\
\hline 30 & 17.69 & 19.74 & 38.34 \\
\hline
\end{tabular}

Table IV: Tabular representation of Mobility based Energy

\begin{tabular}{|c|c|c|c|}
\hline Nodes & $\begin{array}{c}\text { DEPSO- } \\
\text { MCCM }\end{array}$ & EIPBP & AODV \\
\hline 5 & 2.65 & 4.48 & 15.34 \\
\hline 10 & 5.93 & 7.21 & 20.76 \\
\hline 15 & 6.30 & 9.48 & 25.07 \\
\hline 20 & 8.48 & 10.12 & 29.43 \\
\hline 25 & 9.64 & 11.64 & 36.36 \\
\hline 30 & 12.83 & 14.83 & 40.88 \\
\hline
\end{tabular}

Table V: Tabular representation of Mobility based

PDR

\begin{tabular}{|c|c|c|c|}
\hline Nodes & $\begin{array}{c}\text { DEPSO- } \\
\text { MCCM }\end{array}$ & EIPBP & AODV \\
\hline 5 & 94.56 & 92.85 & 84.49 \\
\hline 10 & 92.38 & 90.29 & 74.76 \\
\hline 15 & 91.09 & 87.38 & 72.07 \\
\hline 20 & 90.39 & 83.38 & 68.43 \\
\hline 25 & 58.39 & 80.89 & 65.11 \\
\hline 30 & 83.99 & 78.13 & 60.48 \\
\hline
\end{tabular}

Table II, III, IV, and V shows the tabular representation of the parameters such as PDR, delay, energy, and overhead. These parameters are computed in two factors: the former is mobility-based and latter is pause time-based prediction.

The computed values of the proposed DEPSO-MCCM approach is compared with two existing models known as EIPBP and AODV protocols. The proposed design shows better trade in delay, PDR and energy utilized.

The above mentioned tabular columns are based on the mobility model. Here the simulation is performed in various node placements, i.e., 5, 10, 15, 20, 25 and 30 nodes correspondingly. The control overhead of the proposed method for corresponding nodes is $2.47,8.54,12.49,15.28$, 18.33 and 21.69 respectively.

Similarly, the delay is reduced by $1.34 \mathrm{~s}, 7.38 \mathrm{~s}, 9.67 \mathrm{~s}$, 12.48s, 15.33s and 17.69. As well, for energy consumption is reduced by 2.65, 5.93, 6.30, 8.48, 9.64 and 12.83. Finally, the packet delivery ratio is improved by 94.59, 92.38, 91.09, 90.39, 58.39, 83.99 respectively.

Table VI: Tabular representation of Mobility based Pause time overhead

\begin{tabular}{|c|c|c|c|}
\hline Nodes & $\begin{array}{c}\text { DEPSO- } \\
\text { MCCM }\end{array}$ & EIPBP & AODV \\
\hline 5 & 6.47 & 8.65 & 17.65 \\
\hline 10 & 5.54 & 7.38 & 15.38 \\
\hline 15 & 4.49 & 6.42 & 14.42 \\
\hline 20 & 2.28 & 5.38 & 12.39 \\
\hline 25 & 1.33 & 2.48 & 9.15 \\
\hline 30 & 0.69 & 1.19 & 8.05 \\
\hline
\end{tabular}


Table VII: Tabular representation of Mobility based Pause time delay

\begin{tabular}{|c|c|c|c|}
\hline Nodes & $\begin{array}{c}\text { DEPSO- } \\
\text { MCCM }\end{array}$ & EIPBP & AODV \\
\hline 5 & 3.47 & 5.65 & 13.65 \\
\hline 10 & 2.54 & 4.38 & 11.38 \\
\hline 15 & 1.49 & 3.42 & 10.42 \\
\hline 20 & 0.98 & 1.39 & 8.39 \\
\hline 25 & 0.33 & 1.85 & 7.15 \\
\hline 30 & 0.19 & 0.83 & 7.05 \\
\hline
\end{tabular}

Table VIII: Tabular representation of Mobility based Pause time Energy

\begin{tabular}{|c|c|c|c|}
\hline Nodes & $\begin{array}{c}\text { DEPSO- } \\
\text { MCCM }\end{array}$ & EIPBP & AODV \\
\hline 5 & 6.69 & 9.65 & 18.65 \\
\hline 10 & 6.54 & 7.38 & 16.38 \\
\hline 15 & 5.82 & 6.42 & 15.42 \\
\hline 20 & 4.28 & 5.39 & 12.39 \\
\hline 25 & 3.33 & 4.85 & 11.15 \\
\hline 30 & 2.69 & 3.87 & 10.05 \\
\hline
\end{tabular}

Table IX: Tabular representation of Mobility based Pause time PDR

\begin{tabular}{|c|c|c|c|}
\hline Nodes & $\begin{array}{c}\text { DEPSO- } \\
\text { MCCM }\end{array}$ & EIPBP & AODV \\
\hline 5 & 94.56 & 91.85 & 84.49 \\
\hline 10 & 95.38 & 92.29 & 86.76 \\
\hline 15 & 96.09 & 93.38 & 87.07 \\
\hline 20 & 97.39 & 94.38 & 88.43 \\
\hline 25 & 98.65 & 95.89 & 89.11 \\
\hline 30 & 99.99 & 96.13 & 90.48 \\
\hline
\end{tabular}

Table VI, VII, VIII, and IX show the tabular representation of the parameters such as PDR, delay, energy, and overhead. These parameters are computed in two factors: the former is mobility-based and latter is pause time-based prediction.

The computed values of the proposed DEPSO-MCCM approach is compared with two existing models known as EIPBP and AODV protocols. The proposed design shows better trade in delay, PDR and energy utilized.

The above mentioned tabular columns are based on the pause time model. Here the simulation is performed in various node placements, i.e., 5, 10, 15, 20, 25 and 30 nodes correspondingly. The control overhead of the proposed method for corresponding nodes is 6.47, 5.54, 4.49, 2.28, 1.33 and 0.69 respectively.

Similarly, the delay is reduced by $3.47 \mathrm{~s}, 2.54 \mathrm{~s}, 1.49 \mathrm{~s}$, $0.98 \mathrm{~s}, 0.33 \mathrm{~s}$, and $0.19 \mathrm{~s}$. As well, energy consumption is reduced by $6.69,6.54,5.82,4.28,3.33$, and 2.69. Finally, the packet delivery ratio is improved by $94.56,95.38,96.09$, 97.39, 98.65 and 99.99 respectively.

\section{CONCLUSION}

Here, DEPSO-MCCM model is anticipated. Two foremost constraints of fast-moving nodes are energy and routing. Routing is optimized to save UAVs' by managing transmission range and clustering network effectually. Outcomes of anticipated model are contrasted with two AI algorithms PSO and DE which is for clustering. DEPSOMCCM outcomes compete with cluster count and lifetime,

PDR and delay. DEPSO-MCCM outperforms in cluster construction and energy consumption. In future, high mobility of nodes is accounted to perform routing. DEPSOMCM enhances search capability and to attain superior flexibility in forming nodes cluster. Outcomes demonstrate DEPSO-MCM based clustering algorithm to attain superior performance with number of epochs to acquire fitness function effectually. In the future, hybridization of diverse machine learning algorithm is cast off to achieve $100 \%$ accuracy and network connectivity.

\section{REFERENCES}

1. D. Merwe and A. Engelbrecht, "Data clustering using particle swarm optimization," in Proceedings of the 2003 Congress on Evolutionary Computation. vol. 1, pp. 215-220, 2003.

2. X. Cui, T. Potok, and P. Palathingal, "Document clustering using particle swarm optimization," in Proceedings of 2005 IEEE Swarm Intelligence Symposium, pp. 185-191, 2005.

3. F. Yang, T. Sun, and C. Zhang, "An efficient hybrid data clustering method based on K-harmonic means and particle swarm optimization," Expert Systems with Applications, vol. 36, pp. 9847 9852, 2009.

4. T. Runkler and C. Katz, "Fuzzy clustering by particle swarm optimization" in Proceedings of IEEE International Conference on Fuzzy Systems, pp. 601-608, 2006.

5. M. Omran, A. Salman, and A. Engelbrecht, "Dynamic clustering using particle swarm optimization with application in image segmentation," Pattern Analysis \& Applications, vol. 8, no. 4, pp. 332-344, 2006.

6. S. Das, A. Abraham, and A. Konar, "Automatic kernel clustering with a multi-elitist particle swarm optimization algorithm," Pattern Recognition Letters, vol. 29, pp. 688-699, 2008

7. S. Paterlini and T. Krink, "Differential evolution and particle swarm optimization in partitional clustering," Computational Statistics \& Data Analysis, vol. 50, pp. 1220-1247.

8. S. Das, A. Abraham, and A. Konar, "Automatic clustering using an improved differential evolution algorithm," IEEE Transactions on Systems, Man, and Cybernetics-Part A: Systems and Humans, vol. 38, no. 1, pp. 218-237, 2008.

9. W. Zhang and X. Xie, "DEPSO: Hybrid particle swarm with differential evolution operator," in Proceedings of IEEE International Conference on Systems, Man, and Cybernetics, pp. 3816-3821, 2003.

10. R. Eberhart and Y. Shi, "Particle swarm optimization: developments, applications, and recourses," in Proceedings of the 2001 Congress on Evolutionary Computation, pp. 81-86, 2001.

11. M. S. Fareed, N. Javaid, M. Akbar, S. Rehman, "Optimal Number of Cluster Head Selection for Efficient Distribution of Sources in WSNs", Seventh International Conference on Broadband, Wireless Computing, Communication and Applications, 2012.

12. Hassan EL ALAMI, Abdellah NAJID, "Energy-Efficient Fuzzy Logic Cluster Head selection in Wireless Sensor Networks", IEEE 2016.

13. Choon-Sung Nam, Yong-Ki Ku, Jong-Wan Yoon, and Dong-Ryeol Shin, "Cluster Head Selection for Equal Cluster Size in Wireless Sensor Networks", International Conference on New Trends in Information and Service Science, 2009.

14. Hironori Ando*, Elis Kulla*, Leonard Barolli $\uparrow$, Arjan Durresi $\$$, Fatos Xhafa, "A New Fuzzy-based Cluster-Head Selection System forWSNs", International Conference on Complex, Intelligent, and Software Intensive Systems, 2011.

15. Aniji John, Angaha Rajput and Vinoth Babu K, "Energy Saving Cluster Head Selection in Wireless Sensor Networks for the Internet of Things Applications", International Conference on Communication and Signal Processing, April 6-8, 2017, India

16. Chong Han, Qing Lin, Jian Guo, "A Clustering Algorithm for Heterogeneous Wireless Sensor Networks Based on Solar Energy Supply", Electronics 2018, 7, 103; DOI:10.3390/electronics7070103

17. Pawan Singh Mehra $\Uparrow$, Mohammad Najmud Doja, Bashir Alam, "Fuzzy based enhanced cluster head selection (FBECS) for WSN", Journal of King Saud University - Science, 2018.

18. Achyut Shankar1, Natarajan Jaisankar2, "Hybrid model for securityaware cluster head selection in wireless sensor networks", IET Wireless Sensor Systems, 2018. 
19. Fang Zhu, Junfang Wei, "An Energy-Efficient Routing Protocol Based on Layers and Unequal Clusters in Underwater Wireless Sensor Networks", Hindawi Journal of Sensors Volume 2018, Article ID 5835730, 10 pages https://doi.org/10.1155/2018/5835730

20. Adem Fanos Jemal1, Redwan Hassen Hussen2, Do-Yun Kim2, "Energy-efficient selection of cluster headers in wireless sensor networks", International Journal of Distributed Sensor Networks 2018, Vol. 14(3) _ The Author(s) 2018

21. Thomas BW, White CC III (2007) The dynamic and stochastic shortest path problem with anticipation. Eur J Oper Res 176:836-854

22. Orlin JB, Madduri K, Subramani K, Williamson M (2010) A faster algorithm for the single-source shortest path problem with few distinct positive lengths. J Discr Algorithms 8:189-198

23. Liu W (2010) Uncertain programming models for shortest path problem with uncertain arc length. In: Proceedings of the first international conference on Uncertainty Theory. Urumchi, China, pp $148-153$

24. Hutson KR, Shier DR (2009) Extended dominance and a stochastic shortest path problem. Comput Oper Res 36:584-596

25. Fan YY, Kalaba RE, Moore JE (2005) Shortest paths in stochastic networks with correlated link costs. Comput Math Appl 49:15491564. 